\title{
Medial Axis Approximation and Unstable Flow Complex *
}

\author{
Joachim Giesen ${ }^{\dagger}$ \\ Theoretische Informatik \\ ETH Zürich \\ $\mathrm{CH}-8092$ Zürich \\ giesen@inf.ethz.ch
}

\author{
Edgar A. Ramos \\ Dept. of Computer Science \\ University of Illinois \\ Urbana, IL 61801 \\ eramosn@cs.uiuc.edu
}

\author{
Bardia Sadri \\ Dept. of Computer Science \\ University of Illinois \\ Urbana, IL 61801 \\ sadri@cs.uiuc.edu
}

\begin{abstract}
The medial axis of a shape is known to carry a lot of information about it. In particular a recent result of Lieutier establishes that every bounded open subset of $\mathbb{R}^{n}$ has the same homotopy type as its medial axis. In this paper we provide an algorithm that, given a sufficiently dense but not necessarily uniform sample from the surface of a shape with smooth boundary, computes a core for its medial axis approximation, in form of a piecewise linear cell complex, that captures the topology of the medial axis of the shape. We also provide a natural method to freely augment this core in order to enhance it geometrically all the while maintaining its topological guarantees. The definition of the core and its extension method are based on the steepest ascent flow induced by the distance function to the sample. We also provide a geometric guarantee on the closeness of the core and the actual medial axis.
\end{abstract}

\section{Categories and Subject Descriptors}

F.2.2 [Nonnumerical Algorithms and Problems]: Geometrical problems and computations; I.3.5 [Computational Geometry and Object Modeling]: Curve, surface, solid, and object representations

\section{General Terms}

Theory, Algorithms

\section{Keywords}

Medial Axis Approximation, Gradient Flow, Flow Complex, Stable and Unstable Manifolds

\footnotetext{
${ }^{\dagger}$ Research partially supported by the Swiss National Foundation under the project "Non-linear manifold learning".

*A full version of this paper is available through authors' websites.
}

Permission to make digital or hard copies of all or part of this work for personal or classroom use is granted without fee provided that copies are not made or distributed for profit or commercial advantage and that copies bear this notice and the full citation on the first page. To copy otherwise, to republish, to post on servers or to redistribute to lists, requires prior specific permission and/or a fee.

SCG'06, June 5-7, 2006, Sedona, Arizona, USA.

Copyright 2006 ACM 1-59593-340-9/06/0006 ...\$5.00.

\section{INTRODUCTION}

The medial axis of a bounded open set $S$ in $\mathbb{R}^{n}$ is the set of points in $S$ with at least two closest points in the boundary of $S$. In the following we sometimes refer to bounded open subsets of $\mathbb{R}^{n}$ as shapes. A recent result of Lieutier 13 establishes that any shape and its medial axis have the same topological type, or more precisely, are homotopy equivalent. Consequently, the medial axis can be used to answer topological queries about the shape. Therefore, it can be crucial for a medial axis approximation algorithm to capture the topology of the medial axis when its output is used in applications that make such queries including but not limited to shape analysis, motion planning, and mesh partitioning.

As a geometric object, the medial axis is unstable since small changes in the shape can cause comparatively large changes in its medial axis. This instability of the medial axis bears two consequences. First, it makes the medial axis hard to compute exactly because of numerical instabilities; consequently, exact computation of medial axis has only been attempted for a few limited classes of shapes (see for example [7]). Second, the complete medial axis may be less interesting in practice than an approximation of it which carries the same topological type but is more stable under small perturbations of the shape. Approximations of the medial axis of a shape are often sought with a sample of the boundary of the shape provided as input. Chazal and Lieutier 6] defined the $\lambda$-medial axis, a subset of the medial axis, which has the desired stability, and is guaranteed to have the same homotopy type as the medial axis for suitably small values of the parameter $\lambda$. The largest topologically safe $\lambda$ depends on the shape and can be very small and hard to determine 4]. Finally, the existing algorithm for computing the $\lambda$-medial axis requires a very dense uniform sample of the boundary of the shape 6 .

The $\varepsilon$-sampling theory of Amenta and Bern [1] provides a framework for analysis of algorithms that either reconstruct the boundary of a shape or approximate its medial axis using a sample whose density varies and is proportional to the local size of the features of the boundary of the shape. Several results were obtained in this framework, some of the earliest of which pertaining the medial axis are due to Amenta, Bern, and Eppstein [2], and Boissonnat and Cazals 5], each establishing that a subset of the Voronoi vertices in the Voronoi diagram of the sample points lie close to the medial axis. Later, Amenta, Choi, and Kolluri [3] designed an algorithm that computes an approximation of the medial axis, in shape of a cell complex, that is provably homotopy equivalent to the medial axis of the shape, pro- 
vided that that shape boundary is sampled densely enough. The output of this algorithm tends to be noisy and needs to be cleaned up using heuristics for practical use. The cell complex produced by this algorithm is not contained in the Voronoi 2-skeleton of the input sample, a property sought and considered plausible by many medial axis approximation algorithms due to the fact that the Voronoi 2-skeleton is indeed the medial axis of the sample and that effective filtering of it can often produces good results in practice. Dey and Zhao 9] addressed these shortcomings by designing an algorithm that outputs a sub-complex of the Voronoi diagram as a medial axis approximation. This often geometrically pleasing output is guaranteed to converge to the true medial axis when the sample grows infinitely dense, i.e., when $\varepsilon \rightarrow \infty$, but suffers lacking of any topological guarantees. In fact, despite its celebrated geometric quality, with a poor choice of filtering parameters, the provided output can be highly flawed topologically. The results of the present paper can be used to mend this deficiency.

Our Contribution. Our assumptions and the approach we pursue in this paper also fall into the Amenta-Bern framework. We compute a piece-wise linear cell complex, which we call the core (of the medial axis approximation). This core is extracted from a refinement of the Voronoi complex of the input sample called the unstable flow complex. The idea and the algorithm to compute this complex and the core are derived from the critical point theory of the distance function to the sample points and their induced steepest ascent flow, which we refer to as the discrete flow.

Dey et al. 8. show that the critical points of the distance function to an $\varepsilon$-sampling of the boundary of a shape are naturally separated; each such critical point is either very close to the surface, or oppositely, is very close to the medial axis of the shape. The two classes can be separated algorithmically provided that the sample is sufficiently dense.

In short, the core we compute is the union of the unstable manifolds of the critical points close to the medial axis. The unstable manifold of a critical point consists of all points that can be reached from an infinitesimally small neighborhood of that critical point following the discrete flow. In the full-version of this paper, we characterize the structure of the unstable manifolds and show how to compute them from the Voronoi complex of the sample points in $\mathbb{R}^{3}$. Our main result states that the core and the medial axis are homotopy equivalent at every dimension for dense enough samples.

Capturing the homotopy type of the medial axis is not the only advantage of the core. Once the core is computed, it can be extended "freely" to better capture the geometry of the medial axis while maintaining its homotopy type. To extend the core one picks, using any algorithm at hand, a set of points that approximates a subset of the medial axis of the target shape (see for example 9] for one such algorithm) and adds this set to the computed core along with its flow closure under the discrete flow. We show that as long as the chosen approximating set of points does not get too close to the surface of the shape, adding the closure "recaptures" the topological guarantee of the core. Because of this property, computing the core can be used to augment virtually any medial axis approximation algorithm into a topologically accurate one.

Finally we provide an upper-bound on the rate at which the distance of a point to the medial axis can grow as the point moves along its flow line in the discrete flow. This shows that discrete flow closure of points near the medial axis does not escape the medial axis rapidly. A consequence of such a geometric guarantee is that the core gets closer and closer to the medial axis and is contained in it at the limit as $\varepsilon \rightarrow 0$. Moreover, if the core is extended using a set that converges to the real medial axis when $\varepsilon \rightarrow 0$, such as the Voronoi facets filtered by the algorithm of [9], then the extended core also converges to the real medial axis in Hausdorff distance all the while staying homotopy equivalent to it.

The structure of the paper is as follows. In Section 2 we introduce basic definitions and state some known results. Section 3 establishes several homotopy equivalences, most importantly the homotopy equivalence of the medial axis, the core, and the extended core. Section 4 describes how to provide geometric guarantees for the core and flow closures. Concluding remarks and experimental results are provided in Section 5] As mentioned earlier, the full-version of this paper covers in detail algorithms for computing the unstable flow complex, the core, and flow closures of Voronoi faces.

\section{PRELIMINARIES}

In this section we review some basic definitions as well as some required background material. It is important to notice that although we present all the definitions for $\mathbb{R}^{3}$, all the statements and results of the present section as well as those in sections 3 and 4 generalize to higher dimensional Euclidean spaces.

Basic Notions. For a point $x \in \mathbb{R}^{3}$ and $r>0$, we denote by $B(x, r)$ the open ball of radius $r$ centered at $x$, i.e., $B(x, r)=$ $\left\{y \in \mathbb{R}^{3}:\|x-y\|<r\right\}$, and by $\bar{B}(x, r)$ the closure of $B(x, r)$, i.e., $\bar{B}(x, r)=\left\{y \in \mathbb{R}^{3}:\|x-y\| \leq r\right\}$. We refer to $B(x, r)$ as the $r$-neighborhood of $x$. For $x \in \mathbb{R}^{3}$ and any subset $S \subset \mathbb{R}^{3}$, we define the distance between $x$ and $S$ as $d(x, S)=\inf _{y \in S}\|x-y\|$. By $S^{c}$ we denote the complement of $S$ in $\mathbb{R}^{3}$.

Shape, Surface, and Medial Axis. We consider singlecomponent smooth 2-manifolds without boundary embedded in $\mathbb{R}^{3}$. We call such manifolds surfaces. A surface $\Sigma$ is associated to the two open components of its complement $\mathbb{R}^{3} \backslash \Sigma$ which we refer to as the bounded or inner component $S$, and the unbounded or outer component $S^{*}$. We will refer to the bounded component as the shape enclosed by $\Sigma$ and denote it throughout by $S$. Likewise, in the rest of this paper, $S^{*}$ refers to the unbounded component associated to the considered surface $\Sigma$. The medial axis $M(S)$ of and open set $S$ is the set of all points in $S$ that have at least two closest points in $\partial S$, the boundary of $S$, i.e. $M(S)=\left\{x \in S:\left|A_{S}(x)\right|>1\right\}$, where $A_{S}(x)$ is the set of closest points to $x$ in $\partial S=\Sigma$. Note that since $\Sigma$ is compact, $A_{S}(x)$ is well-defined and non-empty for every $x \in S$. By medial axis $M$ of a surface $\Sigma$, we mean the union of the medial axes of the inner and outer components $S$ and $S^{*}$ associated to $\Sigma$, i.e. $M=M(S) \cup M\left(S^{*}\right)$. We call $M(S)$ and $M\left(S^{*}\right)$ the inner and the outer medial axis medial axis of $\Sigma$ respectively. Thus, $M$ consists of all points in $\mathbb{R}^{3}$ that have at least two closest points in $\Sigma$. 
Feature Size and Surface Samples. By definition, every point of $\mathbb{R}^{3}$ not in $M$ has a unique closest point in $\Sigma$. For any point $x \in \mathbb{R}^{3} \backslash(\Sigma \cup M)$ we denote by $\hat{x}$ the unique closest surface point to $x$, i.e., $\hat{x}=\operatorname{argmin}_{y \in \Sigma}\|x-y\|$, and by $\check{x} \in M$ we denote the center of the medial ball tangent to $\Sigma$ at $\hat{x}$ and at the same side of $\Sigma$ as $x$. The medial feature size is the function $\mu: \mathbb{R}^{3} \backslash(\Sigma \cup M) \rightarrow \mathbb{R} \cup\{\infty\}$ defined as $\mu(x)=\|\hat{x}-\check{x}\|$. The function $f: \Sigma \rightarrow \mathbb{R}, x \mapsto \inf _{y \in M}\|x-y\|$ which assigns to each point in $\Sigma$ its distance to the medial axis $M$, is called the local feature size. Notice that for $x \in$ $\mathbb{R}^{3} \backslash(\Sigma \cup M)$ it always holds that $f(\hat{x}) \leq \mu(x)$. It can also be easily seen that $f$ is 1-Lipschitz. Throughout this paper, we assume that every point $x \in \Sigma$ has non-zero local feature size and that the infimum of the local feature size function over $\Sigma, f_{0}=\inf _{x \in \Sigma} f(x)$, sometimes called the reach of $\Sigma$ in the literature, is bounded away from zero.

For a constant $\varepsilon>0$, a finite sample $P \subset \Sigma$ is called a (relative) $\varepsilon$-sample if for all $x \in \Sigma$, there exists a sample point $p \in P$ withing distance $\varepsilon f(x)$ from $x$.

Distance Functions and Derived Concepts. Given a an open set, $S$ we define the distance function $h_{S}$ induced by $S$ as

$$
h_{S}(x): S \rightarrow \mathbb{R}, \quad x \mapsto d\left(x, S^{c}\right) .
$$

When $S$ is a shape enclosed by a surface $\Sigma, h_{S}(x)=d(x, \Sigma)$ in which we can expand the domain of the function to the entire $\mathbb{R}^{3}$ and denote it by $h_{\Sigma}$. Such distance functions have been broadly studied in the literature (see for example 11]) and are known to carry a great deal of information about the set inducing them and its embedding. For example, $\Sigma$ itself is simply given by $h_{\Sigma}^{-1}(0)$ and the medial axis $M$ of $\Sigma$ turns out to consist of all points at which $h_{\Sigma}$ is not differentiable. The prevalence of information encoded in $h_{\Sigma}$ motivates the use of distance functions induced by discrete samples of $\Sigma$, as computationally manageable "approximations" to it, for extraction of the same kind of information.

The function $h_{S}$ (also $h_{\Sigma}$ and more generally any distance function) is 1-Lipschitz and therefore continuous. However, it is not differentiable everywhere in $S$, i.e., the gradient can be undefined at certain points. As mentioned above, these point constitute $M(S)$. Some of these points are called critical points, namely, the points $x \in S$ that are contained in the convex hull of $A_{S}(x)$. Every other point of $S$ is a regular point. Although the gradient is undefined at many of the regular points in $S$, a unique vector of steepest ascent of $h_{S}$ exists at every such point (see [13]). Let $d_{S}(x)$ be the center of the smallest closed ball containing $A_{S}(x)$ and let $r_{S}(x)$ be the radius of this ball (See Figure 1). We define the flow vector at $x$ (with respect to $S$ ) as

$$
v_{S}(x)=\frac{x-d_{S}(x)}{h_{S}(x)} .
$$

The vector $v_{S}(x)$ agrees with $\nabla h_{S}(x)$ at every point $x \in S$ for which this gradient is defined and extends the gradient of $h_{S}$ everywhere else by determining the direction of steepest ascent for $h_{S}$ [13. The points $x$ for which $v_{S}(x)=0$ are exactly the critical points of $h_{S}$. It can be easily verified using Pythagoras's theorem that

$$
\left\|v_{S}(x)\right\|^{2}=1-\left(\frac{r_{S}(x)}{h_{S}(x)}\right)^{2} .
$$

By showing the convergence of Euler schemes, Lieutier 13

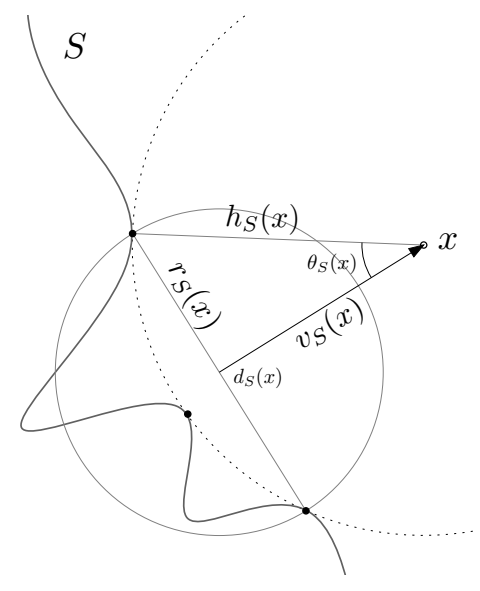

Figure 1: Characterization of the steepest ascent direction for a point $x \in S$. Solid points on the boundary of $S$ represent $A_{S}(x)$.

proved that for any bounded open set $S$, the flow vector field $v_{S}$ as described above can be integrated, to give a map $\phi_{S}: \mathbb{R}^{+} \times S \rightarrow S$ that is continuous in both variables and satisfies for all $x \in S$, (1) $\phi_{S}(0, x)=x$ and (2) $\phi_{S}\left(s, \phi_{S}(t, x)\right)=$ $\phi_{S}(s+t, x)$ for all $s, t \in \mathbb{R}^{+}$. Starting at any point $x \in S$, the flow map $t \mapsto \phi_{S}(t, x)$ defines a continuous path in $S$, called the orbit of $x$ and denoted $\phi_{S}(x)$, when $t$ goes from 0 to $+\infty$. More formally, $\phi_{S}(x)=\left\{y \mid \exists t \in \mathbb{R}^{+}: \phi_{S}(t, x)=y\right\}$. At any point $y \in \phi_{S}(x)$ the flow vector $v_{S}(y)$ determines the direction of the flow at $y$. Lieutier further proved that when $S$ is a bounded open set then for any point $x \in S$ both of the maps $t \mapsto h_{S}\left(\phi_{S}(t, x)\right)$ and $t \mapsto r_{S}\left(\phi_{S}(t, x)\right)$ are increasing and that the former map is continuous and satisfies the following integral equation:

$$
h_{S}\left(\phi_{S}(t, x)\right)=h_{S}(x)+\int_{0}^{t}\left\|v_{S}\left(\phi_{S}(\tau, x)\right)\right\|^{2} d \tau .
$$

It turns out that the critical points of $h_{S}$ are the fixed points of the flow map, i.e., if $c$ is a critical point of $h_{S}$, then $\phi_{S}(t, c)=c$ for all $t \in \mathbb{R}^{+}$.

If the shape $S$ is bounded, then the flow orbit $\phi_{S}(x)$ stays inside $S$ and converges to a critical point $c$ of $h_{S}$ as $t \rightarrow+\infty$. Notice that we consider $c$ to be also in the flow orbit of $x$. For a critical point $c$ of the flow $\phi_{S}$, the set of all points $x$ whose flow orbits converges to $c$ is called the stable manifold of $c$ and is denoted by $\mathcal{S}(c)$. In other words,

$$
\mathcal{S}(c)=\left\{x \mid \phi_{S}(+\infty, x)=c\right\} .
$$

Although there is no flow out of a critical point $c$ of $h_{S}$, it is interesting to know where the points very close to $c$ flow. Some of these points flow into $c$ while other flow away from it. We define the unstable manifold $U(c)$ of a critical point $c$, as the set of all points into which points arbitrarily close to $c$ flow. Formally,

$$
\mathcal{U}(c)=\bigcap_{\varepsilon>0} \bigcup_{y \in B(c, \varepsilon)} \phi_{S}(y) .
$$

With an abuse of terminology, we say that $c$ "flows" into the points of $\mathcal{U}(c)$.

As motivated above, given a sample $P$ of $\Sigma=\partial S$, it is natural to try to approximate the distance function $h_{S}$ by 


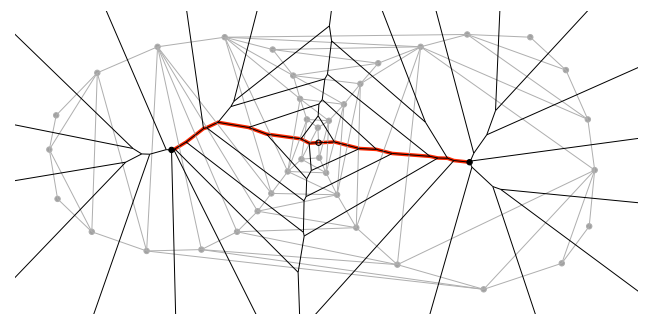

Figure 2: An example of the core in 2D. The sample consists of the gray points. There are three inner medial axis critical points: 2 maxima (in solid black) whose unstable manifolds are singletons made of themselves alone, and a saddle point (hollow) whose unstable manifold is shown in red.

the function $h_{P}: \mathbb{R}^{3} \rightarrow \mathbb{R}, x \mapsto \min _{p \in P}\|x-p\|$, that assigns to each point its distance to the sample $P$ of $\partial S$. The finite set $P$ is the boundary of the open set $P^{c}=\mathbb{R}^{3} \backslash P$ and we can define the distance function and the flow vector for $P^{c}$ as we did for $S$. Since $h_{P c}=h_{P}$, in a slight abuse of notation we will denote both of these distance function with $h_{P}$ and let $A_{P}(x)=\left\{y \in P \mid\|x-y\|=h_{P}(x)\right\}$. We shall also denote the center of the smallest ball containing $A_{P}(x)$ as $d_{P}(x)$ and the associated flow vector field as $v_{P}$. Note that $d_{P}(x)$ is the closest point on the Delaunay face dual to the lowest dimensional Voronoi face that contains $x$. Sometimes $d_{P}(x)$ is referred to as the driver of $x$. Technically speaking, since $P^{c}$ is unbounded, $h_{P}$ has a critical point at infinity. The other critical points of $h_{P}$ can be characterized as the intersection points of Delaunay faces with their dual Voronoi faces 10.

Separation of Critical Points. Dey, et al. 8 observed that if $P$ is an $\varepsilon$-sample of the smooth boundary $\Sigma$ of a shape $S$, then the critical points of the discrete distance function $h_{P}$ cannot reside everywhere in $S$. Rather they have to be either very close to $\Sigma$ or very close to $M$.

THEOREM 1. 8 Let $P$ be an $\varepsilon$-sample of a smooth surface $\Sigma$. Then for every critical point $c$ of $h_{P}$, either $(i)\|c-\hat{c}\| \leq$ $\varepsilon^{2} f(\hat{c})$, or $(i i)\|c-\check{c}\| \leq 2 \varepsilon \mu(c)$.

Thus the critical points of $h_{P}$ can be classified based on whether they are close to $\Sigma$ or close to $M$. We refer to the first class of critical points as surface critical points and to the second class as medial axis critical points. We further subdivide the medial axis critical points $h_{P}$ into two subgroups: inner medial axis critical points are those that are close to $M(S)$ and outer medial axis critical points are those close to $M\left(S^{*}\right)$.

Core. The union of the unstable manifolds of the inner medial axis critical points of $h_{P}$ will play an important role in the present paper we refer to this union as the core of the medial axis approximation (See Figure 2). We will show that for a sufficiently dense sample $P$ of $\Sigma$ this core is homotopy equivalent to the medial axis $M(S)$ of $S$.

Unstable Flow Complex. In general, unstable manifolds of many critical points may intersect. However, in discrete settings, since there are only a finite number of critical points, we can achieve a cell complex decomposition of space by grouping together, as cells, the points of the space that are flowed into from the exactly same "set" of critical points. We define a relation " $\sim$ " on the pairs of points in $\mathbb{R}^{3}$ under which $x \sim y$ if and only if the set of critical points that flow into $x$ coincides with the set of those that flow into $y$, or equivalently, if $x$ is in the unstable manifolds of the same set of critical points as $y$. It is clear that " $~$ " is an equivalence relation. The unstable flow complex induced by a point set $P$, denoted $\mathbf{U}(P)$ (or just $\mathbf{U}$ when $P$ is understood) is the cell complex whose cells are connected components of the subdivision of space into equivalence classes of the " " relation. In the full-version of the paper, we study the structure of this complex more closely in three dimensions. It turns out that the full-dimensional cells of this complex coincide with full-dimensional cells of $\operatorname{Vor}(P)$. The lower dimensional cells of $\mathbf{U}$ introduce a subdivision of the 2-skeleton of $\operatorname{Vor}(P)$. We will see that the core is a sub-complex of $\mathbf{U}$. The importance of these observations is primarily in practice where we desire the output of our algorithms to have a geometric cell-complex structure with no redundancies. Nevertheless, the unstable flow complex of a given set of points can be of independent interest theoretically.

\section{HOMOTOPY EQUIVALENCES}

As in the previous section, we always assume that $\Sigma$ is a smooth manifold with associated inner and outer components $S$ and $S^{*}$. Furthermore, we assume that $P$ is $\varepsilon$ sampling of $\Sigma$. Let $\mathcal{C}$ be the core of the approximation as defined in Section 2, i.e. $\mathcal{C}$ is the union of unstable manifolds of the inner medial axis critical points of the distance function $h_{P}$ contained in $S$. Here we want to show that $\mathrm{C}$ and the medial axis $M(S)$ of $S$ are homotopy equivalent.

Following Lieutier 13 the following criterion is used throughout this paper to prove homotopy equivalence between topological spaces. For the classical definition of homotopy equivalence refer, for example, to 12 .

Proposition 1. Let $X$ and $Y \subseteq X$ be arbitrary sets and let $H:[0,1] \times X \rightarrow X$ be a continuous function on both variables satisfying the following three conditions. (1) $\forall x \in$ $X, H(0, x)=x,(2) \forall x \in X, H(1, x) \in Y$, and $(3) \forall y \in$ $Y, \forall t \in[0,1], H(t, y) \in Y$. Then $X$ and $Y$ have the same homotopy type.

Intuitively, we may interpret the first argument of the map $H$ as time. Using a simple re-parametrization in the first argument, we can replace the interval $[0,1]$ with any interval $[0, T]$ where $T>0$ is a real number. It is important that the time interval considered has finite length. The above criterion for homotopy equivalence between $X$ and $Y$ continuously maps points in $X$ to those in $Y$ during the time interval $[0, T]$. At time 0 , all points in $X$ are mapped to themselves and at time $T$, they have all arrived in $Y$. Notice, the important property that the points in $Y$ stay in $Y$ all the time.

In the following we want to plug in $\phi_{S}$ and $\phi_{P}$ (the flow resulted from integrating $v_{P}$ after circumventing the technical difficulty of unboundedness of $P^{c}$ ) for the map $H$ mentioned above. When distance flow maps are used for $H$, the first condition of Proposition 1 is automatically satisfied since $\phi_{S}(0, x)=x$ for all $x$ (the same holds for $\left.\phi_{P}\right)$. Satisfying 


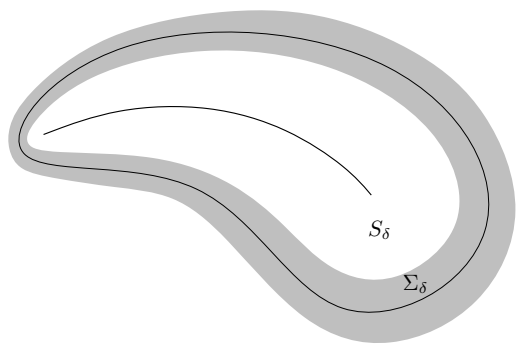

Figure 3: The $\delta$-tubular neighborhood $\Sigma_{\delta}$ (grayed) and the reduced shape $S_{\delta}$.

the second condition when using flows corresponds to proving that every point in $X$ flows into $Y$ in finite time. Finally, the third condition of Proposition 1 to requiring the set $Y$ to be closed for the used flow, meaning that no point of $Y$ flows out of it.

Crucial to the provided homotopy equivalence proofs is the concept of reduced shapes, that we introduce at first.

\subsection{Reduced Shapes}

Reduced Shapes. Let $S$ be a shape whose boundary is a smooth 2-manifold $\Sigma$ and let $0<\delta<1$. The $\delta$-tubular neighborhood $\Sigma_{\delta}$ of $\Sigma$ (See Figure 3 ) is the set

$$
\Sigma_{\delta}=\Sigma \cup\left\{x \in \mathbb{R}^{3} \backslash(\Sigma \cup M):\|x-\hat{x}\|<\delta f(\hat{x})\right\} .
$$

and the $\delta$-reduced shape $S_{\delta}$ is defined as $S_{\delta}=S \backslash \Sigma_{\delta}$. Notice that the definition of $\Sigma_{\delta}$, puts the medial axis $M(S)$ of $S$ into $S_{\delta}$. The following lemma shows that every point of $M(S)$ is in fact an interior point of $S_{\delta}$. Refer to the full-version of the paper for the proof.

Lemma 1. For every $0<\delta<1$, every point of $M(S)$ is an interior point of $S_{\delta}$.

Note, that the previous lemma also implies that every boundary point of $S_{\delta}$ has a unique closest point in $\Sigma$. The following lemma gives a complete characterization of the boundary points of $S_{\delta}$. The proof of this lemma is also provided in the full-version of the paper.

Lemma 2. The boundary of $S_{\delta}$ consists of exactly those points $x \in S \backslash M(S)$ satisfying $\|x-\hat{x}\|=\delta f(\hat{x})$.

\subsection{Homotopy Proofs}

Our proof that the core $\mathcal{C}$ and the medial axis $M(S)$ of $S$ are homotopy equivalent consists of the following two steps.

(1) $S$ and $S_{\delta}$ are homotopy equivalent for $0<\delta<1$.

(2) $S_{2 \varepsilon^{2}}$ and $\mathcal{C}$ are homotopy equivalent for $\varepsilon<0.14$.

The equivalences (1) and (2) together with the homotopy equivalence of $S$ and $M(S) 13$ establish the homotopy equivalence of $M(S)$ and $\mathcal{C}$.

As we mentioned earlier an essential part of each of the homotopy equivalence proofs consists of showing, in correspondence to the second condition of Proposition 1 1 that a considered object is closed under some flow. Trivially, every open set $S$ is closed with respect to the flow $\phi_{S}$ it induces. The medial axis $M(S)$ of an open set $S$ is also closed under $\phi_{S}$. This is because $M(S)$ precisely consists of the points $x \in S$ for which $\left|A_{S}(x)\right|>1$ and consequently $r_{S}(x)>0$. This observation, along with a result of Lieutier 13 that states the map $t \mapsto r_{S}\left(\phi_{S}(t, x)\right)$ is increasing for every $x \in S$, implies that the flow out of every point $x \in M(S)$ stays inside $M(S)$.

Proposition 2. The medial axis $M(S)$ of any open set $S$ is closed under the flow $\phi_{S}$.

The following lemma, due to Lieutier 13], shows that every point $x \in S$ arrives in $M(S)$ under $\phi_{S}$ in finite time.

LEMma 3. 13 Let $\Delta$ be an upper bound for the diameter of an open set $S$. Then for every $x \in S$, we have $\phi_{S}(\Delta, x) \in$ $M(S)$.

The above lemma together with Proposition 2 and the fact that $\phi_{S}(0, x)=x$ for all $x \in S$ fulfill the three requirements of Proposition 1 and therefore imply the following result of Lieutier 13.

Corollary 1. 13 Every bounded open subset $S$ of $\mathbb{R}^{3}$ is homotopy equivalent to its medial axis $M(S)$.

Next we show that the reduced shapes $S_{\delta}$ for $0<\delta<1$ are also closed under the flow $\phi_{S}$. By Proposition 1 this implies that $S$ and $S_{\delta}$ are homotopy equivalent for $0<\delta<1$. The proof is left for the full-version of the paper.

Lemma 4. For any $0<\delta<1$, the reduced shape $S_{\delta}$ is closed under the flow $\phi_{S}$.

Corollary 2. For any $0<\delta<1$, any open set $S$ is homotopy equivalent to its $\delta$-reduced shape $S_{\delta}$.

In order to complete our proof, we need to integrate the vector field $v_{P}$ induced by the same $P$. However, we face a technical difficulty: the result of Lieutier [13] only applies to bounded open sets. Although $P^{c}$ is open, it is not bounded. This problem can be bypassed using a somewhat standard technique. Let $B_{0}=B(c, \Delta / 2)$ to be an open ball of center $c$ and diameter $\Delta$ such that $S \subset B_{0}$ and thus $\Delta$ is an upper bound for diameter of $S$ and let $B_{1}=3 B_{0}=B(c, 3 \Delta / 2)$ be the result of scaling $B_{0}$ three times around its center. Finally let $Q$ be the open set obtained by removing the sample points in $P$ from $B_{1}$, i.e., $Q=B_{1} \backslash P$. Notice that $\partial Q=P \cup \partial B_{1}$. For any point $x$ in $S$, the closest boundary point of $Q$ to $x$ is a point of $P$. This is because every boundary point of $B_{1}$ is at distance strictly greater than $\Delta$ or more from $x$ while there is always a point of $P$ within distance strictly less than $\Delta$ from $x$. This in particular means that the flow induced by $Q$ in $S$, entirely depends on the arrangement of the points in $P$ only and is completely irrelevant to the boundary of $B_{1}$. In other words, the flow lines of the flow $\phi_{Q}$ agree with those of the discrete flow $\phi_{P}$ inside $S$ as described by 10 . As such, in the sequel we shall use the notation $\phi_{P}$ to denote the continuous flow induced by the open set $Q$ inside $B_{0}$. In order to prove an analogue of Lemma 4 for the flow $\phi_{P}$, we first prove following two auxiliary lemmas. The proofs of these lemmas are primarily based on the Lipschitzness of the local feature size function.

Lemma 5. Let $x$ be a point on the boundary of $S_{\delta}$. Any vector $v$ at $x$ that makes an angle $\alpha$ less than $\arctan \left(\frac{1-\delta}{2 \delta}\right)$ with the vector $\check{x}-x$ points into $S_{\delta}$. 


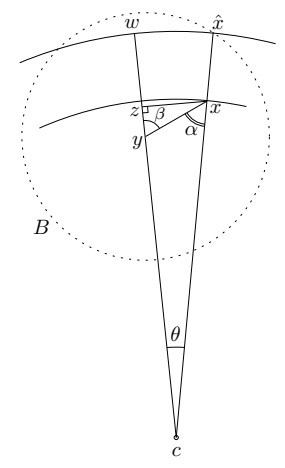

Figure 4: Proof of Lemma 5.

Proof. Let $c$ be the point on the line segment $\hat{x} \check{x}$ at distance $f(\hat{x})$ from $\hat{x}$. Without loss of generality we assume that $v=y-x$, where $y \in S$ is that close to $x$ such that the inner angle $\theta$ of the triangle $x c y$ at $c$ is less than $\pi / 2-\alpha$. Note that by definition $\alpha$ is the inner angle of the triangle $x c y$ at $x$, see Figure 4 Let $B=B(c, f(\hat{x}))$ and let $B^{\prime}=$ $\bar{B}(y,\|\hat{x}-y\|)$. $B$ does not contain any point from $\Sigma$ and $\hat{y} \in B^{\prime}$. Therefore $\hat{y} \in B^{\prime} \backslash B$ and $\|\hat{x}-\hat{y}\| \leq 2 f(\hat{x}) \sin \theta$, which in turn together with the fact that $f$ is 1-Lipschitz implies that

$$
f(\hat{y}) \leq f(\hat{x})(1+2 \sin \theta) .
$$

Let $w$ be the intersection point of the boundary of $B$ with the ray through $y-c$ and let $x^{\prime}$ be the projection of $x$ on this ray. By construction $x^{\prime} \in B$. Our assumption on $\theta$ implies that that on the ray through $c-y$ the point $y$ comes before the point $x^{\prime}$ as seen from $c$. Putting these together we get

$$
\begin{aligned}
\|y-\hat{y}\| & \geq\|y-w\| \\
& =\left\|y-x^{\prime}\right\|+\left\|x^{\prime}-w\right\| \\
& >\left\|y-x^{\prime}\right\|+\|x-\hat{x}\| \\
& =\left\|y-x^{\prime}\right\|+\delta f(\hat{x}) \\
& =\left\|x-x^{\prime}\right\| \cot (\alpha+\theta)+\delta f(\hat{x}) \\
& =(1-\delta) f(\hat{x}) \sin \theta \cot (\alpha+\theta)+\delta f(\hat{x}) .
\end{aligned}
$$

Thus,

$$
\begin{aligned}
\frac{\|y-\hat{y}\|}{f(\hat{y})} & >\frac{(1-\delta) f(\hat{x}) \sin \theta \cot (\alpha+\theta)+\delta f(\hat{x})}{f(\hat{x})(1+2 \sin \theta)} \\
& =\frac{(1-\delta) \sin \theta \cot (\alpha+\theta)+\delta}{1+2 \sin \theta} .
\end{aligned}
$$

In order for $y$ to be in $S_{\delta}$ we want this fraction to be larger than $\delta$. This amounts to $\cot (\alpha+\theta)>2 \delta /(1-\delta)$ or equivalently $\tan (\alpha+\theta)<(1-\delta) / 2 \delta$. Since we assumed $\tan \alpha<$ $(1-\delta) / 2 \delta$ we can find by the continuity of the tangent function $\theta_{0}>0$ such that $\tan (\alpha+\theta)<(1-\delta) /(2 \delta)$ for all $0<\theta<\theta_{0}$. This implies the existence of $\lambda_{0}>0$ such that

$$
(1-\lambda) x+\lambda y=x+\lambda(y-x)=x+\lambda v \in S_{\delta}
$$

for all $0 \leq \lambda<\lambda_{0}$, i.e., the vector $v$ points into $S_{\delta}$ at $x$.

Lemma 6. Let $x$ be a point on the boundary of $S_{\delta}$. The angle $\alpha$ that $v_{P}(x)$ makes with $\check{x}-x$ is bounded by

$$
\arccos \left(\frac{2 \delta(1-\varepsilon-\delta)-\varepsilon^{2}}{2(1-\delta)(\delta+\varepsilon)}\right),
$$

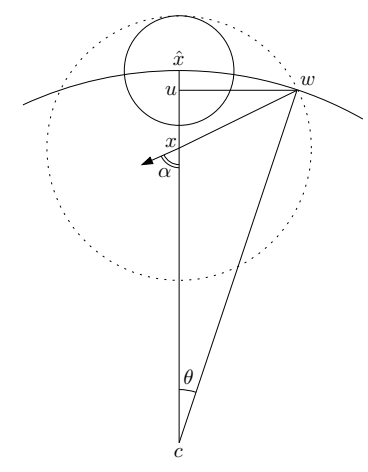

Figure 5: Proof of Lemma 6.

provided that the argument of the arccos is between 0 and 1.

Proof. Let $c$ be the point on the line segment $\hat{x} \check{x}$ at distance $f(\hat{x})$ from $\hat{x}$. Let $B=B(c, f(\hat{x}))$ and let $B^{\prime}=$ $\bar{B}(x,(\delta+\varepsilon) f(\hat{x}))$. The driver $d_{P}(x)$ of $x$ has to be contained in the convex hull of $B^{\prime} \backslash B$. Let $y$ be a point in the intersection of $\partial B$ and $\partial B^{\prime}$. Consider the triangle $c x y$, see Figure 5 The inner angle of this triangle at $x$ is at least $\pi-\alpha$. From the cosine theorem we get

$$
\begin{aligned}
\cos (\pi-\alpha) & \leq \frac{(1-\delta)^{2} f(\hat{x})^{2}+(\delta+\varepsilon)^{2} f(\hat{x})^{2}-f(\hat{x})^{2}}{2(1-\delta)(\delta+\varepsilon) f(\hat{x})^{2}} \\
& =\frac{2 \delta(\delta+\varepsilon-1)+\varepsilon^{2}}{2(1-\delta)(\delta+\varepsilon)}
\end{aligned}
$$

It follows

$$
\cos \alpha \geq \frac{2 \delta(1-\delta-\varepsilon)-\varepsilon^{2}}{2(1-\delta)(\delta+\varepsilon)},
$$

which implies the statement of the lemma.

Now we are ready to prove the analogue of Lemma 4 for the flow $\phi_{P}$.

LemMA 7. The $\delta$-reduced shape $S_{\delta}$ is closed under the flow $\phi_{P}$, respectively, for any $\varepsilon^{2} \leq \delta \leq 10 \varepsilon^{2}$ if $\varepsilon \leq 0.14$.

Proof. Lemmas 5 and 6 imply that for any point $x$ on the boundary of $S_{\delta}$ the vector $v_{P}(x)$ points into $S_{\delta}$ if $\delta \geq \varepsilon^{2}$ and $\varepsilon \leq 0.14$ as can be checked by plugging in the values into the bounds provided by these two lemmas.

Lemma 7 now allows us to prove that the core $\mathrm{C}$ and $S_{\delta}$ are homotopy equivalent. To do so we need the following corollary and lemma.

Corollary 3. For any $\varepsilon^{2} \leq \delta<1$ and $\varepsilon \leq 0.14$, every point of the reduced shape $S_{\delta}$ flows under $\phi_{P}$ into an inner medial axis critical point of $h_{P}$.

Proof. By Lemma 7 every point of $S_{\varepsilon^{2}}$ stays inside $S_{\varepsilon^{2}}$ under $\phi_{P}$ while all surface critical points of $h_{P}$ are in $\Sigma_{\varepsilon^{2}}$ by Theorem 11 Therefore, the flow orbit $\phi_{P}(x)$ of every point $x \in S_{\delta} \subseteq S_{\varepsilon^{2}}$ has to end at a medial axis critical point.

LEMMA 8. For $\varepsilon \leq 0.14$, if $P$ is an $\varepsilon$-sample of $\Sigma$, then there is a constant $T$ such that $\phi_{P}(T, x) \in \mathcal{C}$ for all $x \in S_{2 \varepsilon^{2}}$. 
Proof. Let $\zeta>0$ be the minimum distance between a Delaunay face $D$ induced by $P$ and its dual Voronoi face $V$ that do not intersect. Consider the reduced set $S_{\delta}$ for $\delta=2 \varepsilon^{2}$. By Corollary $3 S_{\delta}$ is closed under the flow $\phi_{P}$. Therefore, every flow line of $\phi_{P}$ in $S_{\delta}$ ends in some inner medial axis critical point of $h_{P}$ in the limit. Consider now any point $x \in S_{\delta} \backslash \mathcal{C}$ and let $V(x)$ be the cell in the Voronoi complex $\operatorname{Vor}(P)$ that contains $x$ and let $D(x)$ be its dual Delaunay cell in the Delaunay complex $\operatorname{Del}(P)$. There are two cases two consider depending on whether $V(x)$ and $D(x)$ intersect.

Case 1. $V(x) \cap D(x)=\emptyset$. In this case, the distance $x-$ $d_{P}(x) \geq \zeta$ and since $h_{P}(x) \leq \Delta$, we get $\left\|v_{P}(x)\right\|=\| x-$ $d_{P}(x) \| / h_{P}(x) \geq \zeta / \Delta$.

Case 2. $V(x) \cap D(x)=\{c\}$, where $c$ is a critical point of $d_{P}$. It can be easily observed that if $c$ is a medial axis critical point, then $V$ is entirely contained in the unstable manifold $\mathcal{U}(c)$ of $c$ and this implies that $x \in \mathcal{C}$, contradicting our choice of $x$. Therefore, $c$ must be a surface critical point and as such $c \in \Sigma_{\varepsilon^{2}}$ while $x \in S_{2 \varepsilon^{2}}$. With a similar argument as the one used in Lemma 2 the open ball of center $c$ and radius $\frac{1-\delta}{1+\delta}\left(2 \varepsilon^{2}-\varepsilon^{2}\right) f(\hat{c})$ is entirely contained in $\Sigma_{2 \varepsilon^{2}}$ and therefore $\|x-c\| \geq \xi=\frac{1-\delta}{1+\delta} \varepsilon^{2} f_{0}$. Thus we get $\left\|v_{P}(x)\right\|=$ $\|x-c\| / h_{P}(x) \geq \xi / \Delta$.

Thus for every point $x \in S_{\delta} \backslash \mathcal{C},\left\|v_{P}(x)\right\| \geq \vartheta$ where $\vartheta=\min \{\xi / \Delta, \zeta / \Delta\}$. If $\phi_{P}(t, x) \notin \mathcal{C}$ for all $t \in\left[0, \Delta / \vartheta^{2}\right]$ we get from Eq. 2

$$
\begin{aligned}
h_{P}\left(\phi_{P}\left(\Delta / \vartheta^{2}, x\right)\right) & \\
& =h_{P}(x)+\int_{0}^{\frac{\Delta}{\vartheta^{2}}}\left\|v_{P}\left(\phi_{P}(\tau, x)\right)\right\|^{2} d \tau \\
& \geq h_{P}(x)+\int_{0}^{\frac{\Delta}{\vartheta^{2}}} \vartheta^{2} d \tau=h_{P}(x)+\Delta .
\end{aligned}
$$

This contradicts the fact that $\Delta$ is an upper bound for the diameter of $S$. Thus if we set $T=\Delta / \vartheta^{2}$, then $\phi_{P}(T, x) \in \mathcal{C}$ for all $x \in S_{\delta} \backslash \mathcal{C}$.

Corollary 4. For $\varepsilon \leq 0.14$, if $P$ is an $\varepsilon$-sample of $\Sigma$, then $\mathrm{C}$ is homotopy equivalent to $S_{2 \varepsilon^{2}}$.

Proof. By definition, $\mathcal{C}$ is closed under the flow $\phi_{P}$. This along with the result of Lemma 8 satisfy the requirements of Proposition 1 implying that $\mathcal{C}$ and $S_{2 \varepsilon^{2}}$ are homotopy equivalent.

Combining the result of the above corollary with that of Corollary 2 gives us the theorem we set out to prove.

TheOREM 2. The shape $S$, its medial axis $M(S)$, and the core $\mathcal{C}$ consisting of the the union of unstable manifolds of inner medial axis critical points of $h_{P}$, are homotopy equivalent.

\subsection{Extending the Core}

As mentioned in the Introduction section, one of the most pleasing properties of the core is its flexibility in being used with other medial axis approximation algorithms. The following Theorem clarifies this statement.

THEOREm 3. Let $W \subset S_{2 \varepsilon^{2}}$ be any set of points and let $\widehat{W}=\left\{x \mid \exists w \in W: x \in \phi_{P}(w)\right\}$. Then $\mathcal{C} \cup \widehat{W}$ is homotopy equivalent to $S$.
Proof. By Lemma 8 all points in $S_{2 \varepsilon^{2}}$ including those in $\widehat{W}$ flow into $\mathcal{C}$ in finite time. On the other hand, by definition $\mathcal{C} \cup \widehat{W}$ is closed for $\phi_{P}$. These are all the requirements needed to invoke Proposition 2 to establish the desired homotopy equivalence between $\mathcal{C} \cup \widehat{W}$ and $S_{2 \varepsilon^{2}}$. By Corollary 2 , the latter is itself homotopy equivalent to $S$.

Computing the flow closure of all the points in $W$ can be computationally difficult, depending on the nature of $W$. However, If $W$ is a sub-complex of the Voronoi complex $\operatorname{Vor}(P)$ (this is for example the case in the algorithm of Dey and Zhao [9]), computing the flow closure of all the points in $W$ can be done in bulk by computing the flow closures of a whole face at a time. The detailed algorithm for computing the flow closure of a Voronoi face can be found in the full-version of this paper. This algorithm immediately can be used for computing the core since the unstable manifold of a critical point is the flow closure of Voronoi faces that contains it.

\section{GEOMETRIC APPROXIMATION}

Geometric fidelity of the core to the real medial axis $M(S)$ of $S$ can be a concern. Although Theorem 1 ensures that the medial axis critical points lie very close to the medial axis, it provides no guarantee for the paths connecting them on their unstable manifolds to enjoy the same closeness. The same concern is valid when we extend the core with a set of points close the medial axis: to guarantee the topology one must include the flow closures of added points but it is not clear that this closure stays close to the medial axis as well.

For a point $x$ in the core $\mathcal{C}$ or any other approximation of the medial axis, the relative approximation error at $x$ can be considered to be the ratio between the distance from $x$ to $M(S)$ and $h_{P}(x)$. One would like to have that the distance to $M(S)$ along a flow line would grow at most linearly with $h_{P}(x)$ as this would correspond to maintaining a constant relative error all along the path. However, proving this, if it is true at all, appears elusive. Nevertheless, in this section we show that if we start from a point $x$ close to the medial axis and follow the flow line $\phi_{P}(x)$, the distance to $M(S)$ along this path grows as a function of $h_{P}$ at a rate slightly super-linear at worst. More precisely we will show that if we scale the space so that $h_{P}(x)$ has unit length, then if $x$ has a medial axis point within distance $O(\sqrt{\varepsilon})$, then every point $y$ in the flow line starting at $x$ will have a medial axis point within distance $O(\sqrt{\varepsilon}) h_{P}(y)^{1+O(\sqrt{\varepsilon})}$. The proof of some technical lemmas are ommited and are availabe in the full-version of the paper.

Lemma 9. For every point $x \in S$ and for every $p \in$ $A_{P}(x),\|x-p\|^{2} \leq h_{S}(x)^{2}+\varepsilon^{2} f(\hat{x})^{2}+\varepsilon^{2} h_{S}(x) f(\hat{x})$.

The driving angle of a point $x$ with respect to the sample $P$ is defined as $\theta_{P}(x):=\angle\left(-v_{P}(x), x-y\right)$ for any $y \in A_{P}(x)$. A critical point has driving angle equal to $\pi / 2$ while points not on the 2-skeleton of $\operatorname{Vor}(P)$ have driving angles equal to 0 . It is not hard to verify that $\cos \theta_{P}(x)=\left\|v_{P}(x)\right\|$.

The following lemma generalizes Theorem 1 although for simplicity it provides a weaker bound for points near the surface. The proof is rather similar to the one given in 8 and is provided in the full-version of the paper. 
Lemma 10. Let $x$ be a point with driving angle $0<\theta \leq$ $\pi / 2$. Then, $x$ is within distance $\frac{2 \varepsilon}{1-\cos \theta} \mu(x)$ from one of $\hat{x}$ or $\check{x}$.

Recall that the driver $d_{P}(x)$ is the same for all points $x$ on the same face of the the Voronoi complex $\operatorname{Vor}(P)$. In fact the flow path $\phi_{P}(x)$ turns exactly when the flow moves from one Voronoi face to another. Consider a point $x \in S$ that lies on the 2-skeleton of $\operatorname{Vor}(P)$, i.e. $\left|A_{P}(x)\right| \geq 2$, and consider a line segment $L$ in the flow orbit $\phi_{P}(x)$. The distance $h_{P}(x)$ monotonically increases along a flow path. We can therefore parametrize this line segment using the distance to the sample set $P$. Let $g: \mathbb{R}^{+} \rightarrow \mathbb{R}^{+}$be a nondecreasing real valued differentiable function and consider for each point $x \in L$, the ball $B_{g}(x)=B\left(x, g\left(h_{P}(x)\right)\right)$. We define the set $D(x)$ as those points on the boundary of $B_{g}(x)$ that are left outside $B_{g}(x)$ when $x$ moves infinitesimally in the direction of $\phi_{P}(x)$. In other words if we take $\eta>0$ such that $\phi_{P}(\eta, x)$ has the same driver as $d_{P}(x)$ (meaning that $\phi_{P}(\eta, x)$ is on the same line segment of $\phi_{P}(x)$ as $\left.x\right)$, then

$$
D(x)=\bigcap_{0<\varepsilon<\eta}\left(\partial B_{g}(x) \backslash \bar{B}_{g}\left(\phi_{P}(\varepsilon, x)\right)\right) .
$$

Lemma 11. $D(x)$ consists of those points $y \in \partial B_{g}(x)$ that satisfy $\angle\left(y-x, d_{P}(x)-x\right) \leq \psi_{0}$ where $\cos \psi_{0}=d g / d h_{P}$ at $x$.

Proof. Let $x^{\prime}=\phi_{P}(t, x)$ be a point on $L$, satisfying $\left\|x-x^{\prime}\right\|=\tau$ where $\tau$ is infinitesimally small. By definition, $A_{P}\left(x^{\prime}\right)=A_{P}(x)$. Let $y$ be any point at distance $g\left(h_{P}(x)\right)$ from $x$ making an angle of $\psi$ with $d_{P}(x)-x$. We have for the distance of $y$ to $x^{\prime}$ :

$$
\begin{aligned}
\left\|y-x^{\prime}\right\|^{2} & =\|y-x\|^{2}+\tau^{2}+2 \tau\|x-y\| \cos \psi \\
& =g\left(h_{P}(x)\right)^{2}+\tau^{2}+2 \tau g\left(h_{P}(x)\right) \cos \psi .
\end{aligned}
$$

For $y$ not to be contained in $B_{g}\left(x^{\prime}\right)$ it must hold that $\| y-$ $x^{\prime} \|>g\left(h_{P}\left(x^{\prime}\right)\right)$, or equivalently:

$$
g\left(h_{P}(x)\right)^{2}+\tau^{2}+2 \tau g\left(h_{P}(x)\right) \cos \psi>g\left(h_{P}\left(x^{\prime}\right)\right)^{2} .
$$

By rearranging we get

$$
\tau+2 g\left(h_{P}(x)\right) \cos \psi>\frac{g\left(h_{P}(x)+\tau\right)^{2}-g\left(h_{P}(x)\right)^{2}}{\tau} .
$$

Taking the limit when $\tau \rightarrow 0$ we get

$$
\begin{aligned}
\lim _{\tau \rightarrow 0} \tau+2 g\left(h_{P}(x)\right) \cos \psi> \\
\lim _{\tau \rightarrow 0} \frac{g\left(h_{P}(x)+\tau\right)^{2}-g\left(h_{P}(x)\right)^{2}}{\tau},
\end{aligned}
$$

which gives

$$
\begin{aligned}
2 g\left(h_{P}(x)\right) \cos \psi & >\frac{d}{d h_{P}}\left(g\left(h_{P}(x)\right)\right)^{2} \\
& =2 g\left(h_{P}(x)\right) \frac{d}{d h_{P}} g\left(h_{P}(x)\right) .
\end{aligned}
$$

Thus $\cos \psi>\cos \psi_{0}$ or $\psi<\psi_{0}$.

Lemma 12. Let $B=B(x, R)$ be a ball empty of sample points with at least one sample point on its boundary and containing at least one medial axis point. Then the ball $B\left(x,\left(1-4 \varepsilon^{2}\right) R\right)$ does not intersect $\Sigma$.
Proof. Let $y \in B \cap \Sigma$. Since $B$ intersects the medial axis, $f(y) \leq 2 R$. Thus there is a sample point within distance $\varepsilon f(y) \leq 2 \varepsilon R$ from $y$. Since $B$ contains no sample points, $y$ must be within distance $2 \varepsilon R$ from $\partial B$. We grow a ball $B^{\prime}$ centered at $x$ until its boundary touches $\Sigma$. Let $R^{\prime}$ be the radius of $B^{\prime}$. By the above argument $R^{\prime} \geq(1-2 \varepsilon) R$. Let $y$ be a point in which $B^{\prime}$ touches $\Sigma$. As indicated above, $f(y) \leq 2 R$. Let $B_{o}$ be the tangent ball of radius $f(y)$ at the opposite side of $\Sigma$ with respect to $x$. With an argument similar to that of Lemma 9 we get for $h_{S}(x)=\|x-y\|$ :

$$
R^{2} \leq h_{S}(x)^{2}+\varepsilon^{2} f(y)^{2}+\varepsilon^{2} h_{S}(x) f(y) .
$$

Using $f(y) \leq 2 R$ we get

$$
R^{2} \leq h_{S}(x)^{2}+4 \varepsilon^{2} R^{2}+2 \varepsilon^{2} R h_{S}(x),
$$

which by rearranging gives the following quadratic inequality for $h_{S}(x)$ :

$$
h_{S}(x)^{2}+2 \varepsilon^{2} R h_{S}(x)-\left(1-4 \varepsilon^{2}\right) R^{2} \geq 0 .
$$

Since $h_{S}(x) \geq(1-2 \varepsilon) R$, the only valid range for $h_{S}(x)$ in the above inequality is

$$
h_{S}(x) \geq \varepsilon^{2} R^{2}+\sqrt{\varepsilon^{4} R^{2}+\left(1-4 \varepsilon^{2}\right) R^{2}} \geq\left(1-4 \varepsilon^{2}\right) R .
$$

TheOREM 4. Let $x_{0} \in S$ be a point with $\left|A_{P}(x)\right| \geq 2$ and $h_{P}\left(x_{0}\right)=h_{0}$, and let $x_{1}=\phi_{P}\left(t_{1}, x_{0}\right)$ be such that for all $0 \leq t \leq t_{1}, \cos \left(\theta_{P}\left(\phi_{P}\left(t, x_{0}\right)\right)\right) \geq c$. If there is a medial axis point within distance $g_{0}$ from $x_{0}$, then there is a medial axis point within distance $g(x)=g_{0}\left(h_{P}\left(x_{1}\right) / x_{0}\right)^{\xi}$ from $x_{1}$, provided that $\xi \geq \frac{1}{c}\left(1+4 \varepsilon^{2} h_{0}^{2} / g_{0}^{2}\right)$.

Proof. We prove the theorem by showing that as $x$ moves along the flow line, there always remains a medial axis point within distance $g_{0}\left(h_{P}(x) / h_{P}\left(x_{0}\right)\right)^{\xi}$ from $x$. We do this by showing that this proposition is maintained when $x$ moves infinitesimally along the flow line. To this end, we first recall that that the ball $B_{g}(x)$ is an open ball by definition. If $B_{g}(x)$ contains a medial axis point $z$, then for any direction vector $v$, there is a small enough real number $\tau>0$, such that the translated ball $B_{g}(x)+\tau v=\left\{y+\tau v \mid y \in B_{g}(x)\right\}$ contains $z$ as well. Since $g$ is increasing, this implies that $z \in B_{g}(x+\tau v)$. In particular this implies by choosing $v=v_{P}(x)$ that if $B_{g}(x)$ contains a medial axis point, so does $B_{g}\left(x+\tau v_{P}(x)\right)$ for $\tau$ sufficiently small.

We thus only need to consider the case where $B_{g}(x)$ contains no medial axis point while its boundary does. Thus, let $z \in \partial B_{g}(x)$ be a medial axis point. By Lemma 11, if $\cos \left(\angle\left(z-x,-v_{P}(x)\right)\right) \leq \frac{d}{d h_{P}} g\left(h_{P}(x)\right)$, then $z$ is contained in $B_{g}\left(x+\tau v_{P}(x)\right)$ for a sufficiently small $\tau>0$ and therefore we have nothing to prove. Thus we only need to consider the case when

$$
\begin{aligned}
\cos \left(\angle\left(z-x,-v_{P}(x)\right)\right) & >\frac{d}{d h_{P}}\left(g\left(h_{P}(x)\right)\right. \\
& =\frac{\xi g_{0}}{h_{P}\left(x_{0}\right)}\left(\frac{h_{P}(x)}{h_{P}\left(x_{0}\right)}\right)^{\xi-1} .
\end{aligned}
$$

We denote this maximum angle by $\psi$. We show that in this case, the flow vector $v(z)$ points to the interior of the ball $B_{g}(x)$. This implies that the flow $\phi(z)$ enters $B_{g}(x)$ and therefore by what we showed above, $B_{g}(x)$ must contain a medial axis, contradicting our choice of $z$.

The ball $B\left(x, h_{P}(x)\right)$ contains no sample points but includes $z$, a medial axis point, and therefore by Lemma 12 


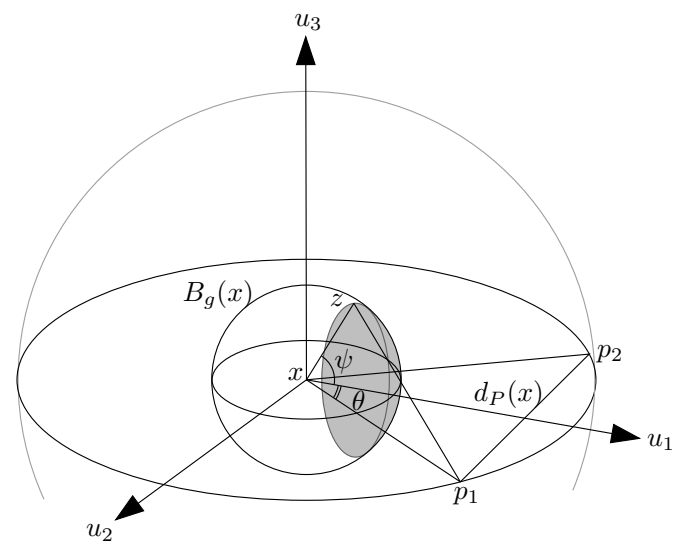

Figure 6: Proof of Theorem 4. The gray cap represents $D(x)$.

the ball $B_{0}=B\left(x,\left(1-4 \varepsilon^{2}\right) h_{P}(x)\right)$ does not intersect $\Sigma$. Consider the plane $\Pi$ tangent to $\partial B_{g}(x)$ at $z$. This plane, intersects the ball $B_{0}$ in a disk of radius

$$
R_{0} \geq \sqrt{\left(\left(1-4 \varepsilon^{2}\right) h_{P}(x)\right)^{2}-g\left(h_{P}(x)\right)^{2}} .
$$

We will show that there are surface points at distance less than $R_{0}$ from $z$, i.e. $h_{S}(z)<R_{0}$. It can be easily observed that any such surface point must lie on the side of $\Pi$ opposite to the one containing $B_{g}(x)$ and therefore $\operatorname{conv}\left(A_{P}(x)\right)$ resides on the side of $\Pi$ opposite to $B_{g}(x)$. Since $d_{P}(x) \in \operatorname{conv}\left(A_{P}(x)\right)$, this implies that $v(x)$ points to the interior of $B_{g}(x)$, as desired. So, all left to show is that $h_{S}(z)<R_{0}$. To prove this, we show that for at least one of the points $y \in A_{P}(x),\|z-y\|<R_{0}$. Since $\left|A_{P}(x)\right| \geq 2$, every plane containing the points $x$ and $d_{P}(x)$ that does not intersect $A_{P}(x)$ must contain at least one point of $A_{P}(x)$ on each side. The maximum distance between $z$ and $A_{P}(x)$ can therefore occur when $\left|A_{P}(x)\right|=2$ and $z$ is on the bisector plane of the segment connecting the two points in $A_{P}(x)$. To find this maximum distance we use a change of coordinates. Denoting the three coordinate directions by $u_{1}, u_{2}$, and $u_{3}$, we put the origin at $x$, the driver $d_{P}(x)$ on the $u_{1}$-axis, the two points $p_{1}, p_{2}$ in $A_{P}(x)$ on the $u_{1} u_{2}$-plane, and $z$ on the $u_{1} u_{3}$-plane (See Figure 6). We can calculate the coordinates of $p_{1}, p_{2}$, and $z$ as follows:

$$
\begin{aligned}
p_{1} & =(h \cos \theta, h \sin \theta, 0), \\
p_{2} & =(h \cos \theta,-h \sin \theta, 0), \\
z & =(g(h) \cos \psi, 0, g(h) \sin \psi),
\end{aligned}
$$

where by $\theta$ we denote the the driving angle $\theta_{P}(x)$ and by $h$ we denote $h_{P}(x)$. Thus we get for the distance between $z$ and $p_{1}$ (same distance between $z$ and $p_{2}$ ):

$$
\begin{aligned}
\| z & -p_{1} \|^{2} \\
& =(h \cos \theta-g(h) \cos \psi)^{2}+(h \sin \theta)^{2}+(g(h) \sin \psi)^{2} \\
& =h^{2}+g(h)^{2}-2 h g(h) \cos \theta \cos \psi
\end{aligned}
$$

Denoting $h_{P}\left(x_{0}\right)$ by $h_{0}$ and $h_{P}(x)$ by $h$ and using the lower bounds for $\cos \psi$ and $\cos \theta$ we get:

$$
\begin{aligned}
\| z & -p_{1} \|^{2} \\
& <h^{2}+g_{0}^{2}\left(\frac{h}{h_{0}}\right)^{2 \xi}-2 c h g_{0}\left(\frac{h}{h_{0}}\right)^{\xi} \frac{\xi g_{0}}{h_{0}}\left(\frac{h}{h_{0}}\right)^{\xi-1} \\
& =h^{2}+(1-2 c \xi) g_{0}^{2}\left(\frac{h}{h_{0}}\right)^{2 \xi}
\end{aligned}
$$

Thus in order for $\left\|z-p_{1}\right\|<R_{0}$, it suffices to have

$$
h^{2}+(1-2 c \xi) g_{0}^{2}\left(\frac{h}{h_{0}}\right)^{2 \xi} \leq\left(1-4 \varepsilon^{2}\right)^{2} h^{2}-g_{0}^{2}\left(\frac{h}{h_{0}}\right)^{2 \xi} \text {. }
$$

Since $\left(1-4 \varepsilon^{2}\right)^{2}>1-8 \varepsilon^{2}$, the above inequality is satisfied when the following one is:

$$
4 \varepsilon^{2} h^{2} \leq(c \xi-1) g_{0}^{2}\left(\frac{h}{h_{0}}\right)^{2 \xi}
$$

Since $h / h_{0} \geq 1$, the above inequality holds if $g_{0} \geq\left(\frac{2 \varepsilon}{\sqrt{c \xi-1}}\right) h_{0}$. This is guaranteed by the bound on $\xi$ prescribed in the statement of the Theorem.

Remark. It may appear at first that when $g_{0}$ goes to zero the above theorem must guarantee a tighter bound. However, the reader must notice that $g_{0}$ also appears in the denominator of the exponent of the given bound. As a result when the flow line starting at a given $x_{0}$ is followed the best bound is not necessarily obtained by using the medial axis point nearest to $x_{0}$. In other words, a larger $g_{0}$ may lead to a better bound on $g$.

Corollary 5. Let $x$ be a point in the 2-skeleton of $\operatorname{Vor}(P)$ such that $\|x-\check{x}\| \leq \frac{2 \sqrt{\varepsilon}}{1-2 \sqrt{\varepsilon}} h_{P}(x)$, then for every point $y$ on the flow path $\phi_{P}(x)$, there is a medial axis point within distance

$$
\frac{2 \sqrt{\varepsilon}}{1-2 \sqrt{\varepsilon}} h_{P}(x)\left(\frac{h_{P}(y)}{h_{P}(x)}\right)^{\xi}
$$

from $y$, where $\xi=1+O(\sqrt{\varepsilon})$.

Proof. We first consider the case where $y$ has a driving angle $\theta=\theta_{P}(y)$ with $\cos \theta \leq 1-\sqrt{\varepsilon}$. By Lemma 10 . $\| y-$ $\check{y} \| \leq 2 \sqrt{\varepsilon} \mu(y)$, or equivalently, $h_{S}(y) \geq(1-2 \sqrt{\varepsilon}) \mu(y)$. Since $h_{P}(y) \geq h_{S}(y)$, we get

$$
\|y-\check{y}\| \leq 2 \sqrt{\varepsilon} \mu(y) \leq \frac{2 \sqrt{\varepsilon}}{1-2 \sqrt{\varepsilon}} h_{S}(y) \leq \frac{2 \sqrt{\varepsilon}}{1-2 \sqrt{\varepsilon}} h_{P}(y) .
$$

When the cosine of the medial angle grows above $1-\sqrt{\varepsilon}$ in a point $y$ along the flow line $\phi_{P}(x)$, by Theorem 4 there always is a point within distance

$$
\frac{2 \sqrt{\varepsilon}}{1-2 \sqrt{\varepsilon}} h_{P}(x)\left(\frac{h_{P}(y)}{h_{P}(x)}\right)^{\xi}
$$

from $y$, where

$$
\xi=\frac{1}{1-\sqrt{\varepsilon}}\left(1+4 \varepsilon^{2} \frac{(1-2 \sqrt{\varepsilon})^{2}}{(2 \sqrt{\varepsilon})^{2}}\right) \leq 1+O(\sqrt{\varepsilon}) .
$$

An immediate consequence of this corollary is that the core and the flow closures converge to being contained in the 

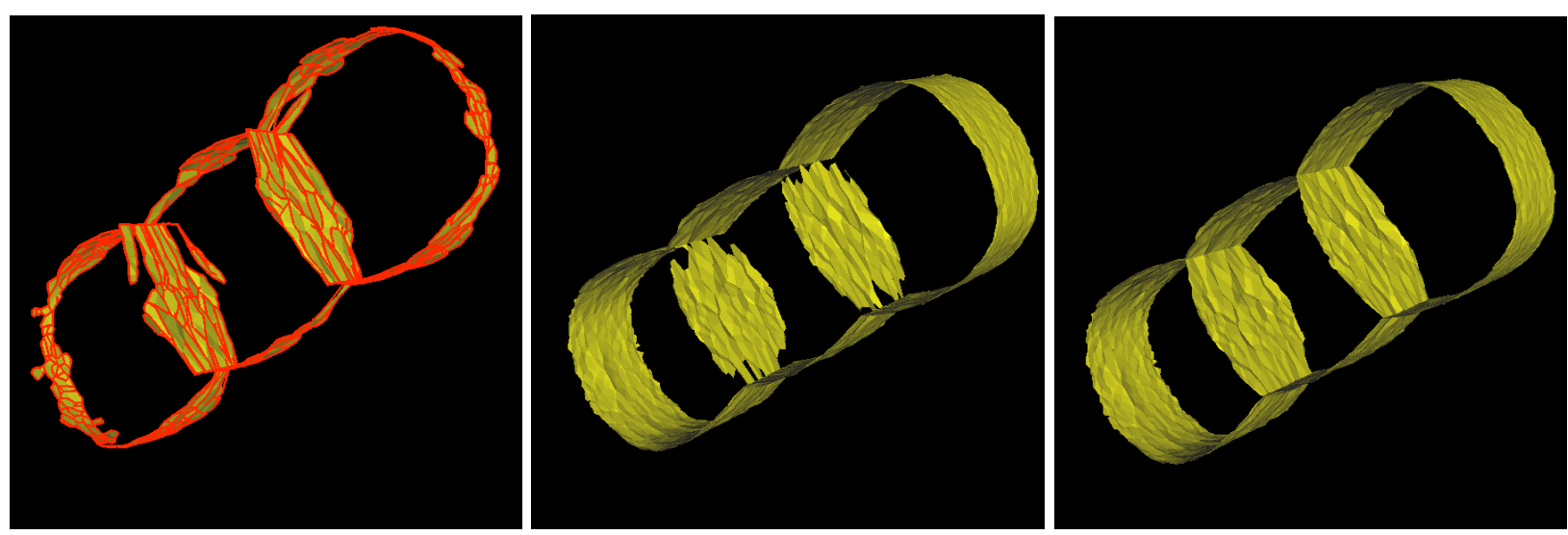

Figure 7: Left: Core computed for the 3-holes model. The red lines are either unstable manifolds of index-2 saddle points or the one dimensional parts (hairs) of index-1 saddle points. Middle: Filtered Voronoi facets based on a condition similar to the angle condition of Dey and Zhao. Right: Extended core. the core, plus the flow closures of the facets in the middle picture.

medial axis as $\varepsilon \rightarrow 0$. As a result, when the core is used and extended using the filtering conditions of [9], the computed approximate medial axis converges to the true medial axis in the limit.

\section{EXPERIMENTS AND CONCLUSION}

We introduced the notion of the "core" as the union of unstable manifolds of medial axis critical points of the distance to and $\varepsilon$-sampling of a surface and established its homotopy equivalence to the sampled shape. We further showed how this core can be safely augmented to provide topological guarantee for any algorithm that geometrically approximates (a subset of) the medial axis. Finally we showed that the computed core and related flow closures do converge to subsets of the medial axis when the input sample grows infinitely dense. A result of the implementation of the unstable flow complex data structure, the core, and the extension of the core using conditions similar to those in 9 is shown in Figure 7. As can be observed, the extension of the core has filled the holes in the junctions of the geometric approximation of the medial axis of the 3-holes model computed by filtering Voronoi facets by a condition similar to the angle condition in 9 .

Acknowledgments. The authors would like to thank Bálint Miklós and Jingyi Jin for the implementation and the presented output pictures.

\section{REFERENCES}

[1] N. Amenta and M. W. Bern. Surface reconstruction by voronoi filtering. Discrete $\mathscr{G}$ Computational Geometry, 22:481-504, 1999.

[2] N. Amenta, M. W. Bern, and D. Eppstein. The crust and the $\beta$-skeleton: Combinatorial curve reconstruction. Graphical Models and Image Processing, 60(2):125-135, 1998.
[3] N. Amenta, S. Choi, and R. K. Kolluri. The power crust, unions of balls, and the medial axis transform. Computational Geometry, 19(2-3):127-153, 2001.

[4] D. Attali, J.-D. Boissonnat, and H. Edelsbrunner. Stability and computation of the medial axis - a state-of-the-art report. Mathematical Foundations of Scientific Visualization, Computer Graphics, and Massive Data Exploration, 2004.

[5] J.-D. Boissonnat and F. Cazals. Smooth surface reconstruction via natural neighbour interpolation of distance functions. Computational Geometry, 22(1-3):185-203, 2002.

[6] F. Chazal and A. Lieutier. The $\lambda$-medial axis. Graphical Models and Image Processing, 67(4):304-331, 2005.

[7] T. Culver, J. Keyser, and D. Manocha. Exact computation of the medial axis of a polyhedron. Computer Aided Geometric Design, 21(1):65-98, 2004.

[8] T. K. Dey, J. Giesen, E. A. Ramos, and B. Sadri. Critical points of the distance to an epsilon-sampling of a surface and flow-complex-based surface reconstruction. In Symposium on Computational Geometry (SCG), pages 218-227, 2005.

[9] T. K. Dey and W. Zhao. Approximating the medial axis from the voronoi diagram with a convergence guarantee. Algorithmica, 38:179-200, 2003.

[10] J. Giesen and M. John. The flow complex: A data structure for geometric modeling. In Symposium on Discrete Algorithms (SODA), pages 285-294, 2003.

[11] K. Grove. Critical point theory for distance functions. Symposia in Pure Mathematics, 54(3):357-385, 1993.

[12] A. Hatcher. Algebraic Topology. Cambridge University Press, 2001.

[13] A. Lieutier. Any bounded open subset of $\mathbb{R}^{n}$ has the same homotopy type as its medial axis. Computer-Aided Design, 36(11):1029-1046, 2004. 